\title{
Linfoma de Burkitt en amígdala palatina de paciente con VIH asociado a tumoración occipital y lesiones osteolíticas en calota
}

\section{Burkitt's lymphoma of the tonsil in HIV patient, associated to occipital tumor and osteolytic lesions in the cranial vault}

\section{Correspondencia}

Giancarlo Pérez Lazo

giancarlo.perez@unmsm.edu.pe

Recibido: 09/01/2017

Arbitrado por pares

Aprobado: 22/02/2017

Citar como: Pérez G, MaqueraAfaray J, Linares S, Castillo $R$. Linfoma de Burkitt en amígdala palatina de paciente con VIH asociado a tumoración occipital y lesiones osteoliticas en calota. Acta Med Peru. 2017;34(1):52-6

\author{
Giancarlo Pérez ${ }^{1,2}$, Julio Maquera-Afaray ${ }^{1,3}$, Sandra Linares ${ }^{4}$, Raúl Castillo ${ }^{1,2}$ \\ 1 Servicio de Medicina Interna-Infectología, Hospital Nacional Guillermo Almenara Irigoyen. Lima, Perú. \\ 2 Universidad Nacional Mayor de San Marcos. Lima, Perú. \\ 3 Universidad Peruana Cayetano Heredia. Lima, Perú. \\ 4 Universidad San Martín de Porres. Lima, Perú.
}

\section{RESUMEN}

El linfoma de Burkitt que afecta a las amígdalas representa el $5 \%$ de los linfomas no Hodgkin primarios extranodales y ocurre usualmente en pacientes con $\mathrm{VIH}$ que presentan valores altos de $\mathrm{CD}_{4}$ y viremia crónica. Presentamos el caso de un varón de 37 años con diagnóstico de VIH menor de 1 año, irregular en la terapia antiretroviral combinada, que presentó una lesión ulcerada, necrótica en amígdala izquierda, y lesiones osteolíticas en calota craneana con compromiso de partes blandas a predominio occipital. En los exámenes auxiliares se halló un recuento de linfocitos $\mathrm{CD}_{4}$ de $50 \mathrm{cel} / \mathrm{mm}^{3}$ y carga viral de $1250000 \mathrm{copias} / \mathrm{mL}$. La biopsia de amígdala fue compatible con Linfoma de Burkitt. El compromiso de calota craneana concomitante a este tumor es raro. Se inició quimioterapia con CODOX-M/IVAC (ciclofosfamida, vincristina, doxorrubicina, metotrexate + citarabina intratecal); sin embargo, la respuesta clínica fue desfavorable, a pesar de la supresión virológica posterior.

Palabras clave: Linfoma de Burkitt; VIH; Calota craneal (fuente: DeCS BIREME).

\section{ABSTRACT}

Burkitt's lymphoma affecting the tonsils represents $5 \%$ of primary extranodal non-Hodgkin lymphomas and it usually occurs in HIV-infected patients with high $\mathrm{CD}_{4}+$ counts and chronic viremia. We report a case of a 37 year-old male diagnosed with HIV infection less than one year ago, who had irregular adherence to HAART. This patient presented with an ulcerated and necrotic lesion in the left tonsil, and he also had osteolytic lesions in the cranial vault, with involvement of the soft tissues, predominantly in the occipital area. His $\mathrm{CD}_{4}+$ count was $50 \mathrm{cells} / \mathrm{mm} 3$ and the viral load (HIV-RNA) was 1'250,000 copies/mL. The biopsy of tonsil was reported as compatible with Burkitt's lymphoma. The patient was started with chemotherapy using CODOX-M/IVAC (cyclophosphamide, vincristine, doxorubicin and methotrexate + intrathecal cytarabine); however, the overall clinical response was poor despite the good virological suppression.

Keywords: Burkitt lymphoma; HIV; Cranial skull (source: MeSH NLM). 


\section{INTRODUCCIÓN}

Los linfomas no Hodgkin (LNH) constituyen un grupo importante y heterogéneo de enfermedades linfoproliferativas; se encuentran dentro de los cánceres asociados a VIH, y se los considera como una enfermedad definitoria de SIDA. Aunque la incidencia de este tipo de linfomas ha disminuido tras la introducción de la terapia antiretroviral combinada ${ }^{[1,2]}$, esta enfermedad aún es un problema a considerar en países con alta frecuencia de casos y diagnóstico tardío ${ }^{[3]}$ como el nuestro.

El linfoma de Burkitt es un subtipo de LNH, infrecuente y agresivo, que contrariamente a las enfermedades oportunistas que afectan al paciente con $\mathrm{VIH}$, afecta fundamentalmente aquellos con recuentos de $\mathrm{CD}_{4}>200 \mathrm{cel} / \mathrm{mm}^{3}{ }^{[4]}$. Además, el compromiso amigdaliano representa aproximadamente solo el $5 \%$ de los LNH primarios extranodales, y la afectación ósea ocurre en estadios tardíos de la enfermedad siendo rara la presentación primaria.

Reportamos un caso de Linfoma de Burkitt de amígdala con lesiones concomitantes en calota craneana en un paciente con infección VIH/SIDA y recuento bajo de $\mathrm{CD}_{4}$.

\section{REPORTE DE CASO}

Paciente varón 37 años, natural del departamento de San Martín y procedente de Lima, con diagnóstico de infección VIH hace 1 año, en terapia antiretroviral combinada (zidovudina, lamivudina y efavirenz), con adherencia irregular y falla virológica a los 6 meses de tratamiento. Hospitalizado por cefalea asociada a tumoración en región occipital de aproximadamente $1,5 \mathrm{~cm}$ de un mes de evolución. Al examen físico, se evidenció en cavidad oral una tumoración dependiente de amígdala izquierda $(2 \times 3 \mathrm{~cm})$ de bordes ulcerados y que desplaza la úvula (Figura 1), que no condicionó odinofagia ni disfagia, y sin adenopatías palpables. No describe síntomas $B$.

En los exámenes por imágenes, la tomografía en región cervicocraneal (Figura 2 y 3 ) mostró una tumoración hipodensa $(32 \times 30 \mathrm{~mm})$ con realce heterogéneo entre pilares amigdalianos

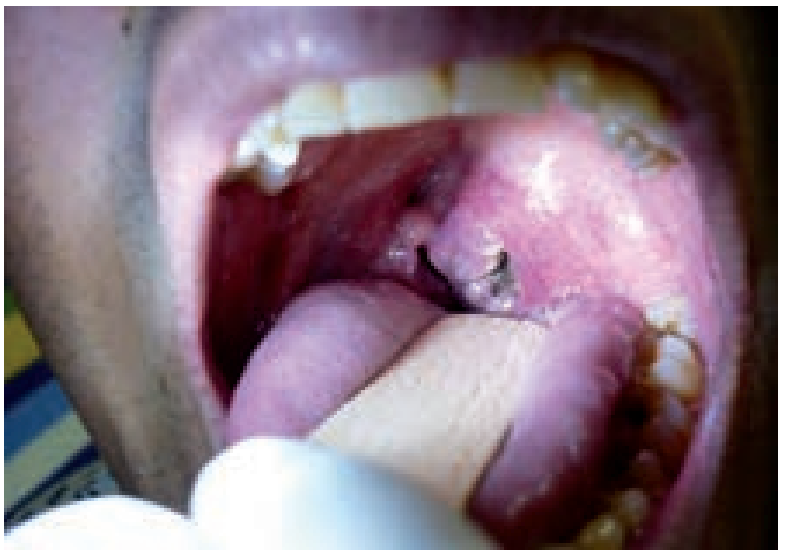

Figura 1. Tumoración dependiente de amígdala izquierda, hipertrófica, ulcerada que desplaza úvula de $2 \times 3 \mathrm{~cm}$.



Figura 2. Tomografía computarizada cervical con contraste, corte sagital. Tumoración hipodensa con realce heterogéneo redondeada de $32 \times 30 \mathrm{~mm}$ en sus diámetros mayores ubicada entre los pilares amigdalianos del lado izquierdo protruida hacia la orofaringe desplazando la úvula. No adenopatías en rango patológico.

izquierdos, que protruye a orofaringe, mostrando una imagen de aspecto polipoide que depende de la pared anterior del seno maxilar derecho; además, se presentan múltiples lesiones osteolíticas en calota con compromiso de partes blandas. En los exámenes de laboratorio de ingreso se encontró un valor de deshidrogenasa láctica (DHL) de $174 \mathrm{UI} / \mathrm{L}, ß_{2}$-microglobulina $3200 \mathrm{mg} / \mathrm{L}$. El hemograma, bioquímica y perfil hepático no mostraron alteraciones. Al ingreso, el recuento de linfocitos $\mathrm{CD}_{4}$ fue de $50 \mathrm{cel} / \mathrm{mm}^{3}$, carga viral para VIH de 1250000 copias/mL.

Se realizó la biopsia de la tumoración de amígdala, que concluyó preliminarmente neoplasia maligna linfoproliferativa con patrón difuso compatible con $\mathrm{LNH}$, con estudios de inmunohistoquímica positivos para $\mathrm{CD}_{45}, \mathrm{CD}_{3}, \mathrm{CD}_{10}$ y CD79a, y el índice de proliferación celular Ki67 fue mayor al 90\%. Los estudios microbiológicos de esta muestra fueron negativos para BAAR, hongos y gérmenes comunes. El diagnóstico histopatológico final fue Linfoma Burkitt, con probable foco primario en amígdala, y lesiones osteolíticas en calota craneal sin confirmación de extensión secundaria. El estudio de aspirado de médula ósea no mostró infiltración por células linfoides atípicas, y las tinciones de Gram, Ziehl Neelsen y PAS fueron negativas. Así mismo, se realizó punción lumbar con resultados negativos para células neoplásicas. No se pudo realizar biopsia del componente subcutáneo en calota para confirmar la extensión de la enfermedad.

Se modificó terapia antiretroviral acorde a genotipo de resistencia a lopinavir/ritonavir, lamivudina, estavudina, y al confirmarse el linfoma de Burkitt se optó por cambiar el inhibidor de proteasa por raltegravir, pues el paciente 


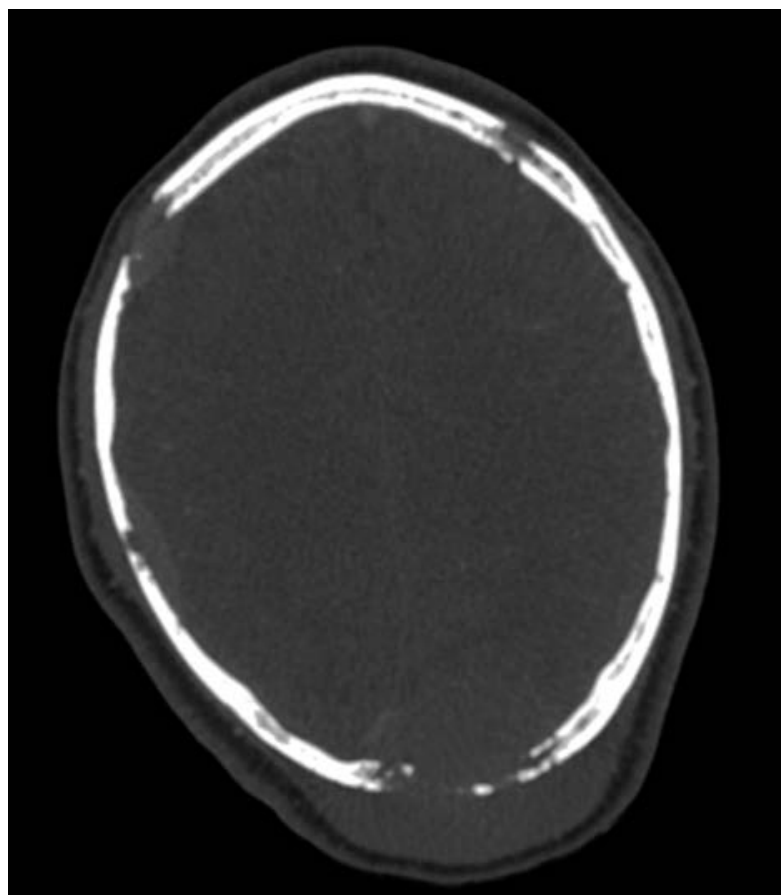

Figura 3. Tomografía computarizada de ventana ósea. Se observa presencia de lesiones osteolíticas en calota craneana de ambos hemisferios cerebrales con formaciones isodensas ovoideas de aspecto tumoral, la mayor a nivel occipital de 60 $\mathrm{mm}$ de diámetro y otras lesiones de similares características isodensas nodulares a nivel parietal derecho de hasta $30 \mathrm{~mm}$ de diámetro que se extiende hacia partes blandas y de localización peridural con compresión del parénquima cerebral subyacente.

recibió quimioterapia con esquema CODOX-M modificado (ciclofosfamida, vincristina, doxorrubicina, y metotrexato + citarabina intratecal). La evolución clínica fue desfavorable, cursando con neutropenia febril profunda y persistente, después del segundo ciclo de quimioterapia, tras lo cual el paciente fallece.

\section{DISCUSIÓN}

El linfoma de Burkitt es una forma de linfoma tipo B que presenta tres variantes clínicas: endémica, esporádica y asociado a $\mathrm{VIH}$. Estas variantes son histológicamente similares e identificadas por su localización geográfica. En la forma asociada a VIH se describe un mayor compromiso de los ganglios linfáticos y la médula ósea. Además, esta entidad tiene el más alto grado de proliferación que cualquier otra neoplasia, con un potencial de doblar su crecimiento en 24 horas y una fracción de crecimiento de $100 \%$ [5].

A pesar que la incidencia de LNH está aumentando en Perú, los casos de Linfoma de Burkitt representan sólo $1 \%$ según últimos datos regionales epidemiológicos ${ }^{[6]}$. En el Perú, Collins et al. reportaron las características clínicas de pacientes VIH con LNH en un centro de referencia y encontraron que el Linfoma de Burkitt representó un $6 \%$ del total de $\operatorname{casos}^{[7]}$.

En el contexto sudamericano, el caso presentado es una entidad rara incluso en población VIH. En comparación a la mayoría de otros linfomas asociados a VIH, los casos de Linfoma de Burkitt en África y Europa no han disminuido en la era de Terapia antiretroviral combinada ${ }^{[8]}$. Son altamente agresivos, y usualmente son diagnosticados en estadios avanzados de la neoplasia. Típicamente afecta pacientes con recuentos de linfocitos $\mathrm{CD}_{4}>200 \mathrm{cel} / \mathrm{mm}^{3}$; sugiriendo que el estado de inmunosupresión no es en sí la única causa de malignidad en $\mathrm{VIH}^{[4]}$.

En adición a la regulación inmune, los linfocitos $T \mathrm{CD}_{4}$ influyen mediante la producción de citoquinas en la supervivencia de células B y T. Este efecto podría explicar la alteración de los centros germinales (en pacientes con infección crónica por $\mathrm{VIH}$ ), áreas en los tejidos linfoides donde las células B proliferan, se diferencian, y aumentan la producción de anticuerpos en respuesta a infecciones. Las células $B$ que residen en los centros germinales, pero no están expuestas a las señales de supervivencia de los linfocitos $\mathrm{TCD}_{4}$ (debido a la eliminación de $\mathrm{CD}_{4}$ por el VIH) están destinadas a sufrir una apoptosis prematura. Si el centro germinal no está presente o está alterado (debido a la falta de $\mathrm{CD}_{4}$ ), el Linfoma de Burkitt no se desarrolla. Esto puede explicar la paradoja del incremento de la incidencia de esta entidad con el aumento de linfocitos $\mathrm{CD}_{4}{ }^{[9]}$.

En el caso presentado se observó una inmunosupresión severa $\left(\mathrm{CD}_{4} 50 \mathrm{cel} / \mathrm{mm}^{3}\right)$; aunque al momento del diagnóstico también había una falla virológica por no adherencia. Se ha documentado que niveles altos de viremia (para VIH, carga viral >10 000 copias/ $\mathrm{mL}$ ), la viremia acumulada y la ausencia de terapia antiretroviral combinada son factores de riesgo para desarrollar $\mathrm{LNH}$, independientemente del nadir de $\mathrm{CD}_{4}$ y de la inmunodeficiencia variable en el tiempo ${ }^{[10]}$.

Otra explicación de que el nivel de $\mathrm{CD}_{4}$ no es suficiente para indicar el potencial maligno es que al momento de la caída de $\mathrm{CD}_{4}$ la neoplasia ya se puede estar desarrollando, indetectable. Además, el nadir de $\mathrm{CD}_{4}$ por sí solo no explica el potencial de subpoblaciones $\mathrm{TCD}_{4}$ en ayudar o regular las respuestas antitumorales ${ }^{[9]}$.

El virus Epstein Barr (VEB) puede estar presente en $50-70 \%$ de los casos de Linfoma de Burkitt, sobre todo la variante plasmocitoide ${ }^{[4,8]}$. Esta asociación no se investigó en el caso presentado por carecer de métodos moleculares en la institución. La interacción entre este virus oncogénico, el VIH y los linfocitos $\mathrm{CD}_{4}$ para promover estas neoplasias permanece aún en investigación activa ${ }^{[9]}$.

La biopsia, a través de la valoración anatomopatológica e inmunohistoquímica, brinda el diagnóstico de confirmación. Nosotros, ante las características de la lesión en amígdala (hipertrófica, ulcerada, necrótica) y la presencia de lesiones múltiples en calota, orientamos el diagnóstico hacía un proceso 
neoproliferativo. En el caso descrito, por las localizaciones descritas, alejamos la posibilidad de que la lesión primaria fuera la occipital pues la amígdala no tiene vías aferentes sino una gran red de vías eferentes que drenan, entre otras, a nivel occipital ${ }^{[1]}$. Se tomó una primera biopsia de la amígdala y estableció que el diagnóstico pudiera ser de naturaleza neoproliferativa (carcinoma epidermoide, sarcoma de Kaposi) o infecciosa (tuberculosis, histoplasmosis, paracocidioidomicosis, y micobacteriosis atípicas) ${ }^{[1]}$.

El linfoma de Burkitt asociado a VIH tiene un inmunofenotipo homogéneo con expresión de $\mathrm{CD}_{20}, \mathrm{CD}_{10}, \mathrm{BCL}-6$, ausencia de $\mathrm{BCL}-2$; y un índice de proliferación (Ki67) mayor a 90\%, como en nuestro caso presentado. En la muestra estudiada, se reportó proliferación de células linfoides atípicas de mediano y gran tamaño y macrófagos con detritus claro. En esta patología se describe la imagen en "cielo estrellado" que corresponde a macrófagos pálidos intercalados con células tumorales [4]; aunque también se puede presentar un pleomorfismo en tamaño y forma de núcleos, citoplasma eccéntrico abundante y núcleo central con nucléolo prominente ${ }^{[8]}$.

En cabeza y cuello, el linfoma de Burkitt afecta con más frecuencia a las amígdalas y luego a las glándulas salivares; respecto al compromiso óseo, la región maxilar superior es la más afectada. En pacientes VIH se presenta usualmente como una masa grande que destruye hueso de región maxilar superior y senos paranasales; también puede comprometer encías y paladar duro en forma de lesiones ulcerativas ${ }^{[12]}$.

De acuerdo a una revisión sistemática, las manifestaciones orales del linfoma de Burkitt fueron edema, dolor, desplazamiento dental y asimetría facial ${ }^{[12]}$. Nuestro paciente estuvo asintomático hasta la aparición de las lesiones en calota, tampoco se presentaron síntomas B, los cuales son reportados en las fases más avanzadas de los linfomas ${ }^{[11]}$.

Ante la presencia de lesiones osteolíticas en calota, se consideraron en el diagnóstico diferencial al mieloma múltiple, linfoma primario de hueso, tuberculosis, y metástasis ${ }^{[3]}$. Si bien el mieloma múltiple presenta lesiones en "sacabocados"; también hay un pico monoclonal sérico ${ }^{[14]}$ que nuestro paciente no tuvo. El compromiso osteolítico en calota secundario a tuberculosis es inusual, casi siempre asociado a tuberculosis pulmonar sistémica, con lesiones focales que simulan a las de mieloma múltiple ${ }^{[15]}$.

Las metástasis craneales son poco frecuentes, y suelen ser secundarias a tumores localizados en tiroides, hígado y pulmón [33]. El linfoma óseo primario es inusual, comprende el $2 \%$ de todos los linfomas y habitualmente se presenta como una lesión única, dolorosa, que se puede asociar a una tumoración de tejidos blandos; siendo los huesos largos como el fémur su ubicación más frecuente ${ }^{[16]}$. No obstante, se han descrito casos cuyo compromiso inicial fue en calota, incluso con lesiones multicéntricas ${ }^{[17]}$.
El compromiso óseo en pacientes con LNH-VIH ocurre en estadios tardíos de la enfermedad y es raro, con escasos reportes en la literatura como foco primario ${ }^{[18]}$. Tanto la densidad en tomografía como intensidad en resonancia magnética de estas lesiones no suelen ser específicas, aunque en los casos reportados se presentan como lesiones hiperdensas, con realce en estudios poscontraste ${ }^{[18]}$.

Adicionalmente a la terapia antiretroviral combinada, se sugieren algunos esquemas quimioterápicos en pacientes con VIH, como el Hyper-CVAD (ciclofosfamida, vincristina, doxorubicina, dexametasona, metotrexate, citarabina), CODOX-M/IVAC; con o sin rituximab, y el DA-EPOCH (dosis ajustadas- etopósido, prednisona, vincristina, ciclofosfamida, doxorubicina) con rituximab ${ }^{[19-21]}$. El esquema CODOX-M/IVAC, usado en nuestro paciente, demostró una respuesta de entre 63$71 \%$ y una supervivencia a los 2 años en $60 \%$, similar a pacientes $\mathrm{VIH}$ negativos y sin aumento de toxicidad ${ }^{[2]}$.

La adición de rituximab a estos esquemas es controversial. Se ha reportado que en pacientes con $\mathrm{CD}_{4}<100 \mathrm{cé} / / \mathrm{mm}^{3}$ hay una menor respuesta, y con recuentos $<50 \mathrm{cel} / \mathrm{mm}^{3}$ mayor riesgo de mortalidad asociada a infecciones ${ }^{[2]}$. Por el contrario, Alwan et al. demostraron que la adición de rituximab al esquema CODOX-M/IVAC concomitante a la terapia antiretroviral combinada no incrementó la toxicidad asociada (infecciones, mucositis, diarrea, falla renal y lisis tumoral), y mejoró significativamente la supervivencia total a los 2 años frente al grupo que no usó rituximab ${ }^{[22]}$.

Los antiretrovirales pueden afectar el metabolismo y compartir toxicidad con los esquemas de quimioterapia; en general, se recomienda no usar zidovudina, cobicistat y ritonavir ${ }^{[21]}$. Se optó la modificación del esquema de rescate usado en el paciente con cambio a raltegravir, pues la combinación lopinavir/ritonavir + vincristina está asociada a complicaciones hematológicas y gastrointestinales. Pese a lograr supresión virológica en este caso, existieron factores vinculados con mal pronóstico para linfoma de Burkitt, como el recuento bajo de $\mathrm{CD}_{4}$, diseminación extranodal, edad mayor a 35 años y estadío clínico III/IV ${ }^{[19]}$. Otros factores descritos son niveles elevados de $\mathrm{DHL}$, y un IPI score (Índice Pronóstico Internacional) modificado a la edad con 2 y 3 puntos ${ }^{[21]}$

En conclusión, aunque no es una patología frecuente, el linfoma de Burkitt debe incluirse en el diagnóstico diferencial de las lesiones en amígdalas en pacientes con $\mathrm{VIH}$, especialmente porque estos pacientes requieren un diagnóstico precoz seguido de un apropiado tratamiento combinado (quimioterapia + terapia antiretroviral) con el objetivo de mejorar el pronóstico de la enfermedad. El diagnóstico tardío y en estadios avanzados de la enfermedad, junto a las posibles complicaciones asociadas a la quimioterapia, hacen que el pronóstico del linfoma de Burkitt sea desfavorable, incluso a pesar de una buena respuesta virológica con la terapia antiretroviral. 


\section{REFERENCIAS BIBLIOGRÁFICAS}

1. Bonnet $F$, Chene G. Evolving epidemiology of malignancies in HIV. Curr Opin Oncol. 2008;20(5):534-40.

2. Corti M, Villafane M, Valergaa $M$, Sforzaa R, Bistmans $A$, Narbaitzc M. Linfoma de Burkitt primario de la cavidad oral en una paciente con sida. Reporte de un caso y revisión de la literatura. Rev Esp Cir Oral Maxilofac. 2015;37(1):44-7.

3. Maquera-Afaray J, Cvetkovic-Vega A, Cárdenas MM, Kälviäinen $\mathrm{H}_{\text {, }}$ Mejia, CR. Diagnóstico tardío y enfermedad avanzada de VIH en pacientes adultos en un hospital de la seguridad social de Perú. Rev Chilena Infectol. 2016;33(Supl 1):20-6.

4. Molyneux E, Rochford R, Griffin B, Newton R, Jackson G, Menon, et al. Burkitt's lymphoma. Lancet. 2012;379(9822):1234-44.

5. Ajila V, Gopakumar R, Hegde S, GS. Intraoral Burkitt's lymphoma in an HIV positive patient. Indian J Sex Transm Dis. 2012;33(2):118-20.

6. Diumenjo MC, Abriata G, Forman D, Sierra MS. The burden of non-Hodgkin lymphoma in Central and South America. Cancer Epidemiol. 2016;44 Suppl 1:S168-S177.

7. Collins JA, HernándezAV, Hidalgo JA, Villena J, Sumire J, Delgado V, et al. High proportion of T-cell systemic non-Hodgkin lymphoma in HIV-infected patients in Lima, Peru. J Acquir Immune Defic Syndr. 2005;40(5):558-64

8. Carbone A, Vaccher E, Gloghini A, Pantanowitz L, Abayomi A, Franceschi $\mathrm{S}$. Diagnosis and management of lymphomas and other cancers in HIV-infected patients. Nat Rev Clin Oncol. 2014; 11(4):223-38.

9. Rubinstein PG, Aboulafia DM, Zloza A. Malignancies in HIV/ AIDS: from epidemiology to therapeutic challenges. AIDS. 2014; $28(4): 453-65$.

10. Achenbach CJ, Buchanan AL, Cole SR, Hou L, Mugavero MJ, Crane $\mathrm{HM}$, et al. HIV viremia and incidence of non-Hodgkin lymphoma in patients successfully treated with antiretroviral therapy. Clin Infect Dis. 2014;58(11):1599-606.

11. Quintana Sanjuás A, Castro Macía O, Hamdan Zavarce M, Pérez Carro-Ríos A, González Guijarro I, Pallas Pallas E, et al. Manifestaciones de los linfomas en el área ORL. Revista Portuguesa de Otorrinolaringologia e Cirurgia Cérvico-Facial. 2010;48(1):25-32
12. Silva TDB, Ferreira CBT, Leite GB, de Menezes Pontes JR, Antunes HS. Oral manifestations of lymphoma: a systematic review. Ecancermedicalscience. 2016;10:665.

13. Iturmendi N, Tomas M, Lillo ME, Tejero C, Relaño MT, Peláez C, et al. Imagen osteolitica en región occipital. REEMO. 2003;12(04):87-8.

14. Reisenbuckler, C. Multiple myeloma and diagnostic imaging. Radiol Technol, 2014;85(4):391-410.

15. Mechán V, Salas A, GarcíaY, Llanos F, Cornejo J, Bringas R. Paciente $\mathrm{VIH}$ positivo con tuberculosis sistémica y lesiones osteolíticas de la calvaria, semejando mieloma múltiple. An Fac Med. 2010;71(3):207-12.

16. Liu M, Liu B, Han F, Song Y. Primary bone lymphoma of the left radius: a case report and related literature review. Eur J Med Res. 2014;(19):19.

17. Graziadio M, Medina N, Amato M, Ardaiz MC, llutovich S, Torino M. Linfoma primario de hueso con afectación multicéntrica. Medicina (Buenos Aires), 2012;72(5):428-30.

18. Thurnher M, Rieger A, Kleibl-Popov C, Schindler E. Malignant lymphoma of the cranial vault in an HIV-positive patient: imaging findings. Eur. Radiol.2001;11(8):1506-9.

19. Basavaraj A, Shinde A, Kulkarni R, Kadam DB, Chugh A. HIV associated Burkitt's lymphoma. J Assoc Physicians India. 2014;62(8):723-7.

20. Bower M, Palfreeman A, Alfa-Wali M, Bunker C, Burns F, Churchill $D$, et al. British HIV Association guidelines for HIV-associated malignancies 2014. HIV Med. 2014;15 Suppl 2:1-92.

21. National Comprehensive Cancer Network. NCCN Clinical Practice Guidelines in Oncology (NCCN Guidelines ${ }^{\circledR}$ ). Non-Hodgkin's Lymphomas Panel. Version 3.2017 (B-cell Lymphomas) [Internet]. Fort Washington, PA: NCCN; $\mathrm{C2}_{2017}$ [citado el 30 de enero de 2017]. Disponible en: https://www.nccn.org/professionals/physician_gls/ pdf/b-cell.pdf

22. Alwan $F$, He A, Montoto $S$, Kassam $S$, Mee M, Burns $F$, et al. Adding rituximab to CODOX-M/IVAC chemotherapy in the treatment of HIV-associated Burkitt lymphoma is safe when used with concurrent combination antiretroviral therapy. AIDS. 2015;29(8):903-10.

\section{Las ediciones anteriores de Acta Médica Peruana están disponibles en:}

\section{www.scielo.org.pe}

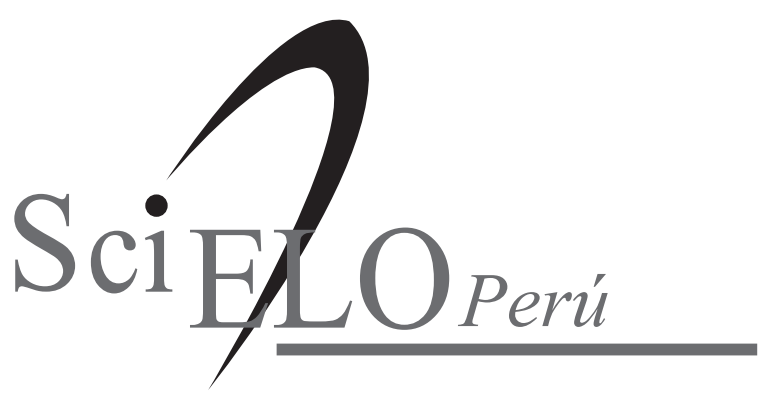

